\title{
Properties of Cardiac Progenitor Cells in the Second Heart Field
}

\author{
Alexandre Francou and Robert G. Kelly
}

\begin{abstract}
Heart tube elongation occurs by progressive addition of cells from pharyngeal mesoderm to the poles of the heart. These progenitor cells, termed the second heart field, contribute to right ventricular and outflow tract myocardium at the arterial pole of the heart and to atrial myocardium at the venous pole. Perturbation of this process results in congenital heart defects. Since the discovery of this progenitor cell population, much has been learned about the signaling pathways and transcription factors regulating second heart field deployment. However, fundamental questions about the molecular and cellular mechanisms underlying heart tube elongation remain. Here we briefly review a selection of recent findings in the area of second heart field biology and discuss the clinical implications of these new studies for our understanding of the etiology of congenital heart defects.
\end{abstract}

\section{Keywords}

Cardiac progenitor cells $\bullet$ Congenital heart defect $\bullet$ Second heart field

\subsection{Introduction}

In 2001 a cardiac progenitor cell population situated in pharyngeal mesoderm was found to give rise to myocardium of the right ventricle and outflow tract. In the intervening period, it has become apparent that this progenitor cell population (1) is part of a larger population of cardiac progenitor cells termed the second heart field (SHF) that also contributes to atrial myocardium, (2) corresponds to a genetic

\footnotetext{
A. Francou • R.G. Kelly $(\bowtie)$

University of Aix-Marseilles, Developmental Biology Institute of Marseilles, CNRS UMR 7288, Campus de Luminy Case 907, 13288 Marseilles Cedex 9, France

e-mail: Robert.Kelly@univ-amu.fr
}

T. Nakanishi et al. (eds.), Etiology and Morphogenesis of Congenital Heart Disease, DOI 10.1007/978-4-431-54628-3_23 
lineage distinct from the first heart field (FHF) that gives rise to the cardiac crescent and left ventricle, and (3) is progressively deployed in the pharyngeal region during embryonic heart development through the activity of multiple signaling pathways and transcription factors. Of particular relevance is the discovery that perturbation of SHF development in animal models and human patients results in a spectrum of congenital heart defects (CHD) affecting the poles of the heart, including conotruncal and atrial septal defects. Details of these features of SHF development have been documented in a series of recent reviews $[1,2]$. Here we will discuss a selection of recently published studies that impact on our understanding of second heart field biology, with focus on mechanistic insights into the etiology of CHD.

\subsection{Demarcating the First and Second Heart Fields and Their Contributions to the Heart}

The distinction between the FHF and SHF has been controversial, despite evidence from clonal analysis and genetic lineage studies that these progenitor populations correspond to separate lineages $[1,2]$. Further support for a two-lineage model of heart development has been provided by a study involving Boolean modeling of gene regulatory networks in heart development [3]. This work identified two stable states corresponding to the FHF and SHF and suggests that the differences between these states are hardwired into the signaling and transcription factor interactions operative in the early embryo. This modeling approach highlights the temporal distinction between the FHF and SHF and can be used to predict gene function, providing an important step toward integrated understanding of regulatory networks during early heart development.

The discovery that Hcn4, encoding a nucleotide-gated channel protein, is expressed in the FHF, in a complementary pattern to the SHF gene Isll, has reinforced the concept that the vertebrate heart is built from distinct progenitor cell populations [4, 5]. An inducible Cre allele of $\mathrm{Hcn} 4$ has allowed evaluation of the contribution of the FHF to the definitive heart. Unlike the SHF, which contains multipotent cardiovascular progenitor cells, FHF derivatives appear to be restricted to myocardium [4]. Interestingly, the two lineages contribute differently and in a complementary manner to different components of the cardiac conduction system [5]. In particular, the right bundle branch and majority of the right Purkinje fiber system have a SHF origin. Similar observations have recently been made based on retrospective clonal analysis and regionalized transgene expression data, supporting dual contributions of the FHF and SHF to the conduction system [6]. These findings are of relevance in understanding the origins of arrhythmias resulting from perturbed development of particular segments of the conduction axis. 


\subsection{New Insights into the Role and Regulation of Noncanonical Wnt Signaling in the Second Heart Field and the Origins of Conotruncal CHD}

Continued proliferation and delayed differentiation are defining properties of the SHF; indeed, separation of the sites of proliferation and differentiation provides a mechanism allowing rapid growth of the embryonic heart. The role and regulation of the noncanonical Wnt ligands Wnt5 and Wnt11 in these processes have been the focus of a number of recent studies. While both ligands were known to be individually required for outflow tract morphogenesis, they have now been shown to be co-required for SHF development [7], by downregulating the canonical Wnt pathway during myocardial differentiation and activating noncanonical Wnt planar cell polarity (PCP) signaling. Wnt5 is expressed before Wnt11 in SHF cells in the posterior dorsal pericardial wall and has been shown to be directly activated by the DiGeorge syndrome candidate gene $T b x 1$, a key regulator of proliferation and differentiation in the SHF [8]. The severity of conotruncal defects in Wnt5a null mice is increased in the presence of a $T b x 1$ null allele, while double mutant embryos lack the right ventricle and outflow tract and die at midgestation. TBX1 directly regulates $W n t 5 a$ through interaction with BAF60a, a progenitor cell-specific component of the BAF chromatin remodeling complex, as well as the histone methyltransferase SETD7, interactions shown to be necessary for activation of a number of TBX1 transcriptional targets in the SHF [8]. Tbxl itself is regulated by the activity of a histone acetyltransferase, MOZ, loss of function of which partially phenocopies DiGeorge syndrome [9]. The intersection of Tbxl with chromatin regulators is highly significant given the recent finding that de novo mutations in genes encoding such molecules are overrepresented in human CHD patients [10].

In support of a role for Tbx 1 upstream of noncanonical Wnt signaling, wnt $11 \mathrm{r}$ is downregulated in $t b x l$ mutant zebrafish; heart looping and differentiation defects in the absence of $t b x l$ can be partially rescued by ectopic wnt11r or a wnt11r target gene encoding a cell adhesion molecule, alcama [11]. Altered cell shape in $t b x l$ mutant fish hearts suggests that noncanonical Wnt signaling regulates cardiomyocyte cell polarity downstream of tbx1 [11]. Whether Tbx1 and noncanonical Wnt signaling also regulate cell polarity in the SHF remains to be seen. SHF and conotruncal development is impaired in mice lacking Dvl1 and Dvl2, regulators of both canonical Wnt signaling and the noncanonical Wnt PCP pathway [12]. In this study, the PCP signaling function of $D v l$ genes was shown to be specifically required in the SHF lineage and the cardiac phenotype to resemble that of embryos lacking the core PCP gene Vangl2; furthermore, Wnt5a and Vangl2 interact to increase the severity of outflow tract defects [12]. Loss of PCP gene function results in disorganization of progenitor cells in the posterior dorsal pericardial wall, potentially resulting in impaired SHF deployment toward the outflow tract and conotruncal anomalies.

Among the last myocardial derivatives of the SHF to be added to the elongating heart tube is future subpulmonary myocardium, a cell population specifically affected in Tbxl mutant embryos [13]. Asymmetric addition of SHF progenitor 
cells giving rise to subpulmonary myocardium continues on the left side of the outflow tract up until embryonic day 12.5 in the mouse; furthermore, this late contribution drives rotation of the outflow tract, positioning subpulmonary myocardium on the ventral side of the heart and aligning the ascending aorta with the left ventricle [14]. Failure of this process, termed the "pulmonary push," results in outflow tract alignment defects such as double outlet right ventricle. Underdevelopment of subpulmonary myocardium has been implicated in the etiology of tetralogy of Fallot [15], and further analysis of the regulation of future subpulmonary myocardium is an important step toward deciphering the etiology of CHD.

\subsection{Involvement of the Second Heart Field in Atrial and Atrioventricular Septal Defects}

At the venous pole of the heart, SHF cells contribute to the dorsal mesenchymal protrusion that bridges the atrioventricular cushions and primary atrial septum. Failure of proliferation or precocious differentiation of these cells results in primum atrial septal defects when canonical Wnt or hedgehog signaling, respectively, is compromised [1, 2]. BMP signaling has now been shown to promote the proliferation of DMP progenitor cells, in contrast to the pro-differentiation role of this signaling pathway during SHF addition at the arterial pole [16]. Isl1, Wnt2, and the transcription factor Gli1 are expressed in this region of the SHF and have been shown to identify a multipotent cardiopulmonary progenitor cell population that contributes not only to the venous pole of the heart but also to diverse pulmonary lineages, including smooth muscle and endothelium [17]. The development of these cells that ensure the vascular connection between the heart and lung is coordinated by hedgehog signaling from future pulmonary endoderm. The Holt-Oram syndrome gene Tbx5 also operates in this posterior component of the SHF and is required for normal atrial septation through regulating proliferation and hedgehog signal reception by direct activation of cell cycle progression genes such as $C d k 6$ and the hedgehog signaling component Gas1 [18]. Genetic and retrospective lineage studies have revealed that venous pole and future subpulmonary myocardium are clonally related, suggesting that a population of common SHF progenitor cells segregates to the arterial and venous pole of the heart [19]. Where such common progenitor cells are located and how their segregation to the poles is regulated remains unknown.

\subsection{Future Directions and Clinical Implications}

The importance of perturbed SHF development in the etiology of common forms of CHD affecting both poles of the heart is now clear. The studies discussed here provide new insights into the underlying mechanisms, although much remains to be learnt about the regulatory interactions between signaling pathways and 
transcription factors controlling cellular properties of the SHF and how different regions of the heart are prepatterned and segregate within the progenitor cell population. Future research will address these questions in the context of dynamic heart tube elongation. The human SHF, as defined by ISl1 expression, coincides with that observed in avian and mouse embryos [20], and thus findings from these models are directly relevant to a better understanding of the origins of CHD in man. Furthermore, insights into how the differentiation of cardiac progenitor cells is controlled are essential for future regenerative therapies.

Acknowledgments Supported by the Fondation pour la Recherche Médicale, Agence National pour la Recherche and Association Française contre les Myopathies.

Open Access This chapter is distributed under the terms of the Creative Commons AttributionNoncommercial 2.5 License (http://creativecommons.org/licenses/by-nc/2.5/) which permits any noncommercial use, distribution, and reproduction in any medium, provided the original author(s) and source are credited.

The images or other third party material in this chapter are included in the work's Creative Commons license, unless indicated otherwise in the credit line; if such material is not included in the work's Creative Commons license and the respective action is not permitted by statutory regulation, users will need to obtain permission from the license holder to duplicate, adapt or reproduce the material.

\section{References}

1. Vincent SD, Buckingham ME. How to make a heart: the origin and regulation of cardiac progenitor cells. Curr Top Dev Biol. 2010;90:1-41.

2. Kelly RG. The second heart field. Curr Top Dev Biol. 2012;100:33-65.

3. Herrmann F, Gross A, Zhou D, et al. A boolean model of the cardiac gene regulatory network determining first and second heart field identity. PLoS One. 2012;7:e46798.

4. Spater D, Abramczuk MK, Buac K, et al. A HCN4+ cardiomyogenic progenitor derived from the first heart field and human pluripotent stem cells. Nat Cell Biol. 2013;15:1098-106.

5. Liang X, Wang G, Lin L, et al. HCN4 dynamically marks the first heart field and conduction system precursors. Circ Res. 2013;113:399-407.

6. Miquerol L, Bellon A, Moreno N, et al. Resolving cell lineage contributions to the ventricular conduction system with a Cx40-GFP allele: a dual contribution of the first and second heart fields. Dev Dyn. 2013;242:665-77.

7. Cohen ED, Miller MF, Wang Z, et al. Wnt5a and Wnt11 are essential for second heart field progenitor development. Development. 2012;139:1931-40.

8. Chen L, Fulcoli FG, Ferrentino R, et al. Transcriptional control in cardiac progenitors: Tbx1 interacts with the BAF chromatin remodeling complex and regulates Wnt5a. PLoS Genet. 2012;8:e1002571.

9. Voss AK, Vanyai HK, Collin C, et al. MOZ regulates the Tbx1 locus, and Moz mutation partially phenocopies DiGeorge syndrome. Dev Cell. 2012;23:652-63.

10. Zaidi S, Choi M, Wakimoto $\mathrm{H}$, et al. De novo mutations in histone-modifying genes in congenital heart disease. Nature. 2013;498:220-3.

11. Choudhry P, Trede NS. DiGeorge syndrome gene tbx 1 functions through wnt11r to regulate heart looping and differentiation. PLoS One. 2013;8:e58145.

12. Sinha T, Wang B, Evans S, et al. Disheveled mediated planar cell polarity signaling is required in the second heart field lineage for outflow tract morphogenesis. Dev Biol. 2012;370:135-44. 
13. Theveniau-Ruissy M, Dandonneau M, Mesbah K, et al. The del22q11.2 candidate gene Tbx1 controls regional outflow tract identity and coronary artery patterning. Circ Res. 2008;103:142-8.

14. Scherptong RW, Jongbloed MR, Wisse LJ, et al. Morphogenesis of outflow tract rotation during cardiac development: the pulmonary push concept. Dev Dyn. 2012;241:1413-22.

15. Van Praagh R. The first Stella van Praagh memorial lecture: the history and anatomy of tetralogy of Fallot. Semin Thorac Cardiovasc Surg Pediatr Card Surg Ann. 2009:19-38.

16. Briggs LE, Phelps AL, Brown E, et al. Expression of the BMP receptor Alk3 in the second heart field is essential for development of the dorsal mesenchymal protrusion and atrioventricular septation. Circ Res. 2013;112:1420-32.

17. Peng T, Tian Y, Boogerd CJ, et al. Coordination of heart and lung co-development by a multipotent cardiopulmonary progenitor. Nature. 2013;500:589-92.

18. Xie L, Hoffmann AD, Burnicka-Turek O, et al. Tbx5-hedgehog molecular networks are essential in the second heart field for atrial septation. Dev Cell. 2012;23:280-91.

19. Lescroart F, Mohun T, Meilhac SM, et al. Lineage tree for the venous pole of the heart: clonal analysis clarifies controversial genealogy based on genetic tracing. Circ Res. 2012;111:1313-22.

20. Sizarov A, Ya J, de Boer BA, et al. Formation of the building plan of the human heart: morphogenesis, growth, and differentiation. Circulation. 2011;123:1125-35. 